2011

\title{
Electronic structures and bonding of graphyne sheet and its $\mathrm{BN}$ analog
}

Jian Zhou

Peking University

Kun Lv

Peking University

Qian Wang

Peking University, Virginia Commonwealth University

See next page for additional authors

Follow this and additional works at: http://scholarscompass.vcu.edu/phys_pubs

Part of the Physics Commons

Zhou, J., Lv, K., Wang, Q. et al. Electronic structures and bonding of graphyne sheet and its $\mathrm{BN}$ analog. The Journal of Chemical Physics, 134, 174701 (2011). Copyright (C) 2011 AIP Publishing LLC.

\section{Downloaded from}

http://scholarscompass.vcu.edu/phys_pubs/102

This Article is brought to you for free and open access by the Dept. of Physics at VCU Scholars Compass. It has been accepted for inclusion in Physics Publications by an authorized administrator of VCU Scholars Compass. For more information, please contact libcompass@vcu.edu. 
Authors

Jian Zhou, Kun Lv, Qian Wang, X. S. Chen, Qiang Sun, and Puru Jena 


\title{
Electronic structures and bonding of graphyne sheet and its BN analog
}

\author{
Jian Zhou, ${ }^{1}$ Kun Lv, ${ }^{2}$ Qian Wang, ${ }^{2,3}$ X. S. Chen, ${ }^{4}$ Qiang Sun, ${ }^{1,2,3, a)}$ and Puru Jena ${ }^{3}$ \\ ${ }^{1}$ Department of Advanced Materials and Nanotechnology, Peking University, Beijing 100871, China \\ ${ }^{2}$ Center for Applied Physics and Technology, Peking University, Beijing 100871, China \\ ${ }^{3}$ Department of Physics, Virginia Commonwealth University, Richmond, Virginia 23284, USA \\ ${ }^{4}$ Shanghai Institute of Technical Physics, Chinese Academy of Science, Shanghai 200083, China
}

(Received 1 February 2011; accepted 1 April 2011; published online 2 May 2011)

\begin{abstract}
Using density functional theory and generalized gradient approximation for exchange and correlation, we present theoretical analysis of the electronic structure of recently synthesized graphyne and its boron nitride analog (labeled as $\mathrm{BN}$-yne). The former is composed of hexagonal carbon rings joined by $\mathrm{C}$-chains, while the latter is composed of hexagonal $\mathrm{BN}$ rings joined by $\mathrm{C}$-chains. We have explored the nature of bonding and energy band structure of these unique systems characterized by $s p$ and $s p^{2}$ bonding. Both graphyne and $\mathrm{BN}-\mathrm{yne}$ are found to be direct bandgap semiconductors. The bandgap can be modulated by changing the size of hexagonal ring and the length of carbon chain, providing more flexibilities of energy band engineering for device applications. The present study sheds theoretical insight on better understanding of the properties of the novel carbon-based 2D structures beyond the graphene sheet. (C) 2011 American Institute of Physics. [doi:10.1063/1.3583476]
\end{abstract}

Carbon, the element responsible for life on earth, has many unique properties brought about by its ability to form diverse $s p, s p^{2}$, and $s p^{3}$ bonding. In organic systems, carbon atoms form a variety of structures ranging from linear chain to square, pentagonal, and hexagonal rings. In molecules, such as coronene, five hexagonal rings of $\mathrm{C}$ surround a pentagonal ring resulting in a buckled structure. However, in condensed matter systems for a long time only two allotropes of carbon have been known. These are diamond and graphite, respectively, characterized by $s p^{3}$ and $s p^{2}$ bonding. The discovery of $\mathrm{C}_{60}$ fullerene in 1985 fundamentally changed this paradigm. New concepts of carbon bonding in condensed matter systems emerged opening the door to the synthesis of other allotropic forms of carbon. These include carbon nanotubes, graphene, ${ }^{1,2}$ and several graphene-based structures, such as graphene nanoribbons, ${ }^{3}$ single atomic C-chain, ${ }^{4,5}$ and porous graphene sheet. ${ }^{6,7}$ Unusual mechanical, electronic, and transport properties of these nanocarbon systems and their many technological applications ranging from drugs to synthetic materials have led to considerable interest in the study of C-based materials.

Very recently, a novel two-dimensional (2D) periodic framework (graphdiyne), composed of $s p$ and $s p^{2}$ mixed C atoms has been synthesized ${ }^{8}$ through cross-coupling reaction using hexaethynylbenzene on copper surface. This series of structure is beyond graphene sheet and has been highlighted in Nature recently. ${ }^{9}$ To exploit its potential technological applications, it is important to investigate the stability and electronic properties of this new 2D structure family. Currently, there are some theoretical works examining the properties of graphyne and its related structures such as nanotubes. ${ }^{10-13}$ However, the electronic structures as well as bandgap modulations of such 2D frameworks have not been examined carefully, such as through electron distribution's point of view and

\footnotetext{
a)Electronic mail: sunqiang@pku.edu.cn.
}

the comparisons with pristine graphene sheet have not been made yet.

In this study, we present theoretical study of the structure and electronic properties of 2D graphyne and their structural analogs involving $\mathrm{BN}$ rings (BN-yne). The latter is composed of $\mathrm{BN}$ hexagonal rings linked by $\mathrm{C}$-chains and have never been examined till now. We note that $\mathrm{BN}$ sheet and its hybrid sheets with graphene have already been successfully synthesized. ${ }^{14-16}$ Using calculations based on density functional theory within generalized gradient approximation, we examine the geometric and electronic properties of graphyne and $\mathrm{BN}-\mathrm{yne}$ as a function of C-chain length. We find that both the systems are semiconductors with direct bandgaps. The C-chains between hexagonal rings have alternative single $\mathrm{C}-\mathrm{C}$ and triple $\mathrm{C} \equiv \mathrm{C}$ bonds, confirmed by bond length and electron distribution analyses. Furthermore, while the bandgap of graphyne is insensitive to the length of C-chains, it decreases monotonically in BN-yne. These bandgap modulation characters, as far as we know, have not been studied and presented in previous theoretical calculations.

Our calculations are based on generalized gradient approximation with Perdew-Burke-Ernzerhof exchange correlation functional, ${ }^{17}$ as implemented in DMOL3 package. ${ }^{18,19}$ We used effective core potential with double numerical polarized basis set. The orbital cutoff is set globally with the value of $3.7 \AA$ for graphyne and $4.1 \AA$ for BN-yne. Vacuum space of $18 \AA$ in $z$ direction is used in order to avoid interactions between two images. We use Monkhorst-Pack special k point meshes $9 \times 9 \times 1$ to represent the reciprocal space. ${ }^{20}$ Geometric structures were relaxed without any symmetric constraints. Convergence of total energy, Hellman-Feynman force, and maximum displacement are set to be $1 \times 10^{-4} \mathrm{eV}, 0.01 \mathrm{eV} / \AA$, and $0.005 \AA$, respectively. All of the computed geometric and electronic properties have been recalculated with Vienna $a b$ initio simulation package (VASP) (Refs. 21 and 22) using projected augmented wave approach, which gave very similar 


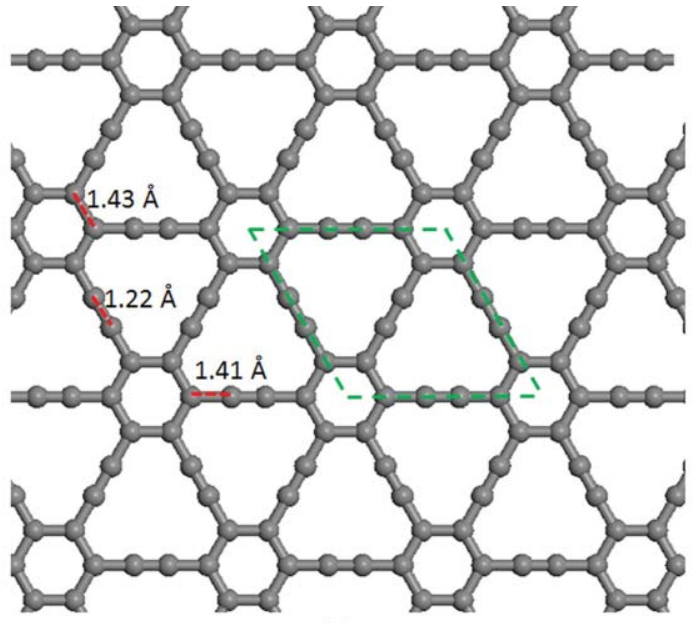

(a)
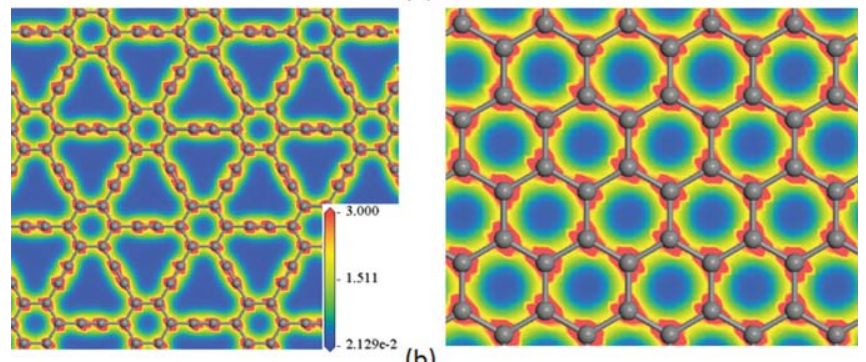

(b)
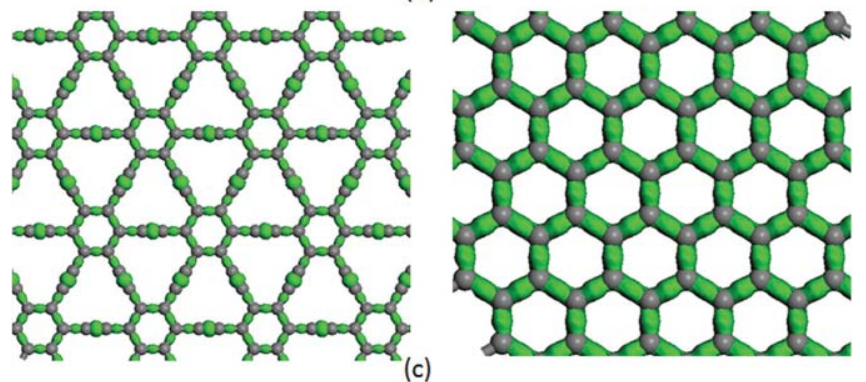

FIG. 1. (a) Geometric structure of optimized graphyne. The unit cell is represented with the green dashed lines. (b) Contour plots of total electron density of graphyne (left panel) and graphene (right panel) for comparison. (c) Isosurface of deformation electron density with value of 0.25 electron $/ \AA^{3}$ of graphyne (left panel) and graphene (right panel).

results from DMOL3 code. The accuracy of the simulation procedure has also been established in our previous studies. $^{23-25}$

First, we optimized the geometric structure of graphyne [Fig. 1(a)]. After relaxation, the system retains its planar structure, consistent with experimental observation. As shown in the figure, the distance between two nearest neighbor hexagonal rings are found to be $6.90 \AA$, which is also the cell constant simulating the rhombus unit cell. The $\mathrm{C}-\mathrm{C}$ bond lengths can be used to determine the type of bond. Generally speaking, the length of single $\sigma$ bond is $\sim 1.47 \AA$, while the double $(\sigma+\pi)$ and triple $(\sigma+2 \pi)$ bonds shrink to $\sim 1.38 \AA$ and $\sim 1.21 \AA$, respectively. Note that here $\pi$ indicates localized $\pi$ orbital. When a delocalized big $\pi$ orbital takes part in bonding the corresponding bond length will be elongated a little. In hexagonal $\mathrm{C}$ rings of graphyne, the bond length is $1.43 \AA$, which lies between single and double bonds, corresponding to $(\sigma+$ big $\pi)$ binding, similar to that of a pristine graphene sheet, $1.42 \AA$. The bond length in the inner C-chain is calculated to be $1.22 \AA$, close to the $\mathrm{C} \equiv \mathrm{C}$ triple bond. Hence, due to the tetravalent nature of $\mathrm{C}$, the bond between the hexagonal ring and the $\mathrm{C}$-chain should be a single $\mathrm{C}-\mathrm{C} \sigma$ bond. However, the bond length is $1.41 \AA$ which is smaller than a pure single $\mathrm{C}-\mathrm{C}$ bond, namely, $1.47 \AA$. This can be understood from electrostatic attraction between two $\mathrm{C}$ sites and will be discussed in the following. The above results are quantitatively consistent with previous results. ${ }^{10}$

The above analyses can be further confirmed from total electron density and deformation electron density, as shown in Figs. 1(b) and 1(c), where data for pristine graphene are also given for comparison. The deformation electron density is defined as the difference between total density and those of isolated atoms. On the $\mathrm{C}$-chains, electrons are accumulated on the bonds of central two $\mathrm{C}$ atoms, demonstrating $\mathrm{C} \equiv \mathrm{C}$ bond character. In the hexagonal rings, both total electron and deformation electron distributions of graphyne are quite similar to those of pristine graphene sheet, consistent with $(\sigma+\operatorname{big} \pi)$ bonding behavior. Mulliken charge analysis shows that each hexagonal ring receives altogether 0.384 electron ( 0.064 per $\mathrm{C}$ atom) from neighboring $\mathrm{C}$-chains. This charge transfer is due to different electron affinities of the hexagonal $\mathrm{C}$ ring and the $\mathrm{C}$-chain. Therefore, the bond between hexagonal ring and the $\mathrm{C}$-chain has some ionic character, which can shrink the bond length compared to that in pure single $\mathrm{C}-\mathrm{C} \sigma$ bond. We also find that the electron distribution in graphyne with all even numbered $\mathrm{C}$-chains is same as described above, namely, alternating single $\mathrm{C}-\mathrm{C}$ and triple $\mathrm{C} \equiv \mathrm{C}$ bond. We have also calculated the structure of graphyne with odd numbered C-chains, and observed serious structural distortions. This is due to the two unpaired electrons in the odd-number $\mathrm{C}$-chains making the chain more reactive. ${ }^{26}$ Therefore, we will not discuss these cases in the present study.

In order to investigate the mechanisms of semiconducting property of graphyne, we calculated the wave functions at valence-band maximum (VBM) and conduction-band minimum (CBM). Both VBM and CBM are found to be located at $\bar{M}$ point [Fig. 2(a)] with bandgap of $0.47 \mathrm{eV}$. According to reciprocal space folding scheme, it is easy to show that for a $(2 \times 2)$ supercell of graphyne the VBM and CBM will be both at the point, which has been confirmed by our calculations (not shown here). Therefore, in the following, we discuss electron behavior of frontier energy band at point of (2 $\times 2$ ) supercell, which can reflect the VBM and CBM properties. In Figs. 2(b) and 2(c), the wave functions of highest occupied (HO) energy state and lowest unoccupied (LU) energy state are plotted. Green (light grey) and red (dark grey) isosurface represent positive and negative signs of wave function, respectively, indicating opposite phases. The HO state is highly localized on the $\pi$ orbital of hexagonal rings and $\mathrm{C} \equiv \mathrm{C}$ bonds. On the other hand, the LU state is located at the $\sigma^{*}$ bond between the ring and $\mathrm{C}$-chain. These highly localized $\pi$ $\mathrm{HO}$ and $\sigma^{*} \mathrm{LU}$ states of graphyne are different from delocalized $\pi$ and $\pi *$ pristine graphene sheet, which makes graphyne semiconducting and graphene metallic.

Next, in order to further understand how the properties of the C-chain can be affected in different bonding environments, we consider the analogous system of $\mathrm{BN}$ sheet, 


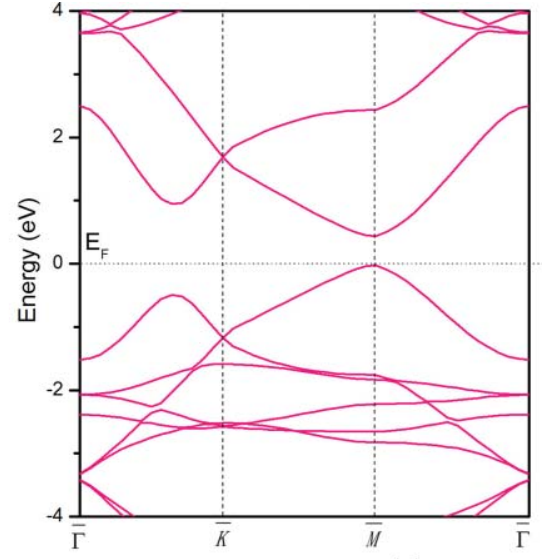

(a)
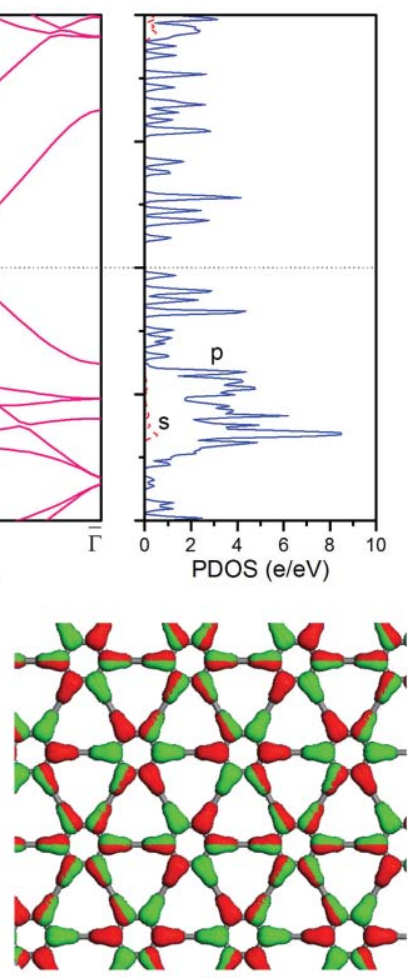

(c) (b)

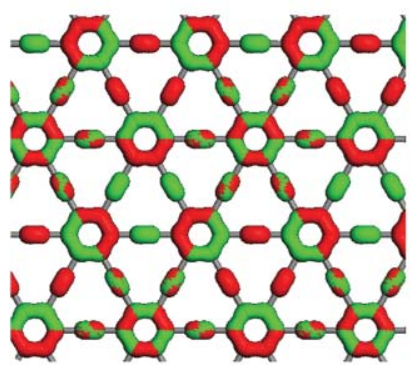

FIG. 2. (a) Band structure and corresponding partial density of states (PDOS) of graphyne, where $(0,0,0), \bar{K}(-1 / 3,2 / 3,0)$, and $\bar{M}(0,1 / 2,0)$ represent high symmetric points in the reciprocal space. (b) Wave function of highest occupied states and (c) lowest unoccupied states at point in $(2 \times 2)$ supercell of graphyne, with isosurface of 0.03 electron $/ \AA^{3}$. Green (light grey) and red (dark grey) colors represent positive and negative signs, respectively.

BN-yne [Fig. 3(a)]. This was inspired by the recent experiment on hybrid $\mathrm{BN}$ and $\mathrm{C}$ sheet. ${ }^{12}$ Geometric relaxation reveals that the unit cell lattice is $7.00 \AA$, a little larger than that of graphyne, namely, $6.90 \AA$. All bond lengths are denoted in Fig. 3(a). We can see that the $\mathrm{C}-\mathrm{C}$ bond length is again $1.22 \AA$, which shows $\mathrm{C} \equiv \mathrm{C}$ triple bond character as discussed above. The bond length between $\mathrm{C}$ and $\mathrm{B}(\mathrm{N})$ is 1.50 (1.35) $\AA$, due to different atomic radius of $\mathrm{B}$ and $\mathrm{N}$ atoms. In the $\mathrm{BN}$ hexagon, the $\mathrm{B}-\mathrm{N}$ bond is optimized to be $1.47 \AA$, which agrees with that of pristine $\mathrm{BN}$ sheet, namely, $1.452 \AA{ }^{27}$ Electron total density and deformation density of $\mathrm{BN}$-yne are also plotted [Figs. 3(b) and 3(c)] and are compared with pristine BN sheet. We see that the charges are mainly accumulated around $\mathrm{N}$ atoms and $\mathrm{C} \equiv \mathrm{C}$ bond. The $\mathrm{C}-\mathrm{B}$ and $\mathrm{C}-\mathrm{N}$ bonds possess some ionic character, with electrons mainly localized around $\mathrm{C}$ and $\mathrm{N}$ atoms, respectively. Electron density distribution in the $\mathrm{BN}$ hexagonal ring is quite similar to that in pristine $\mathrm{BN}$ sheet. Therefore, the B-N bonds again have ionic character. Mulliken charge analysis reveals that $\mathrm{N}$ atoms receive 0.538 electrons from neighboring $\mathrm{C}$ and $\mathrm{B}$, while $\mathrm{B}$ atoms donate 0.454 electrons. Accordingly, $\mathrm{C}$ atoms linked by $\mathrm{N}$ and $\mathrm{B}$ carry 0.249 and -0.162 electrons, respectively. Recall that in pristine $\mathrm{BN}$ sheet, $\mathrm{N}(\mathrm{B})$ atoms receive (lose) 0.603 electrons, larger than those in BN-yne. This is due to larger difference in electron affinities between $\mathrm{B}$ and $\mathrm{N}$ in pristine $\mathrm{BN}$ sheet compared to that between $\mathrm{B}-\mathrm{C}$ and $\mathrm{C}-\mathrm{N}$ in $\mathrm{BN}-\mathrm{yne}$.

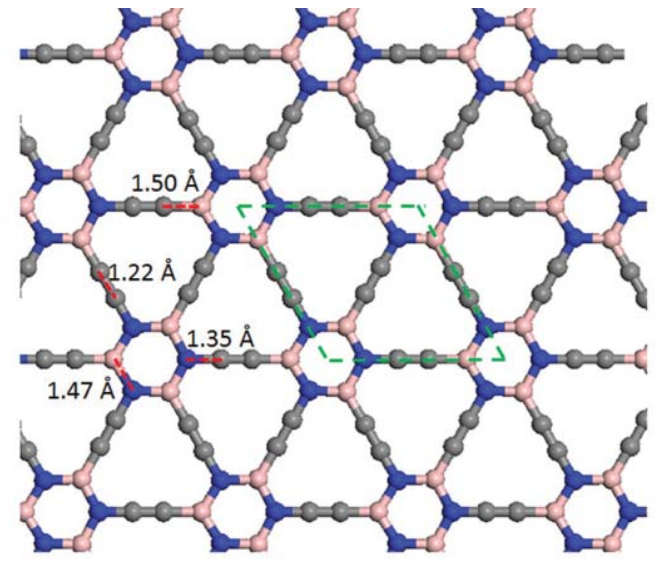

(a)

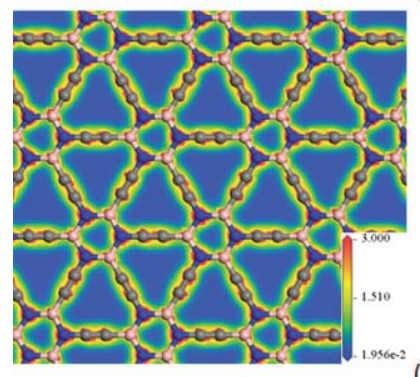

(b)
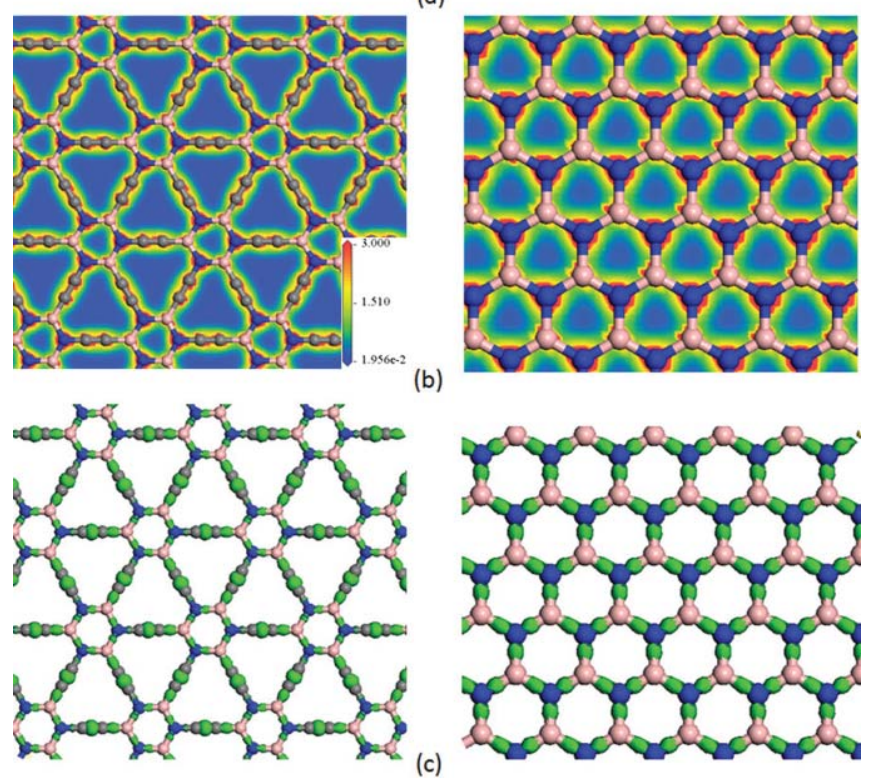

FIG. 3. Same legends as Fig. 1 for BN-yne.

Band structure and partial density of states (PDOS) of $\mathrm{BN}-y n e$ are given in Fig. 4(a). Similar to graphyne, the BNyne is also semiconducting with VBM and CBM both located at $\bar{M}$ point of reciprocal space. The bandgap is $2.65 \mathrm{eV}$ which is smaller than that of pristine $\mathrm{BN}$ sheet, $4.64 \mathrm{eV}^{23}$ The PDOS shows that this bandgap is contributed from $p$ orbital, same as that in pristine $\mathrm{BN}$ sheet. Wave functions of $\mathrm{HO}$ and $\mathrm{LU}$ at point of $(2 \times 2)$ supercell are plotted in Figs. 4(b) and 4(c). It is found that the $\mathrm{HO}$ is contributed from $p$ orbitals of negatively charged $\mathrm{N}$ atoms and $\mathrm{C} \equiv \mathrm{C}$ bond, while $\mathrm{LU}$ originates from $p$ orbitals of positively charged $\mathrm{B}$ atoms and $\mathrm{C}$ atoms of $\mathrm{C}-\mathrm{N}$ bonds. This character is similar to that of pristine $\mathrm{BN}$ sheet, which has $\mathrm{HO}$ and LU contributed from $\mathrm{N}$ and $\mathrm{B}$ atoms, respectively. ${ }^{27}$

Finally we discuss the modulation of bandgap in graphyne- and BN-yne-based structures, where the nodes (hexagonal rings) and linkers (C-chains) are the basic building blocks. It is easy to imagine that the bandgap of the system can be tuned by changing the node size and/or the length of the linker, providing more flexibility as compared to the bandgap modulations in pristine graphene ${ }^{23}$ and $\mathrm{BN}$ sheet $^{25}$ for nanoelectronics and nanodevice applications. Our 


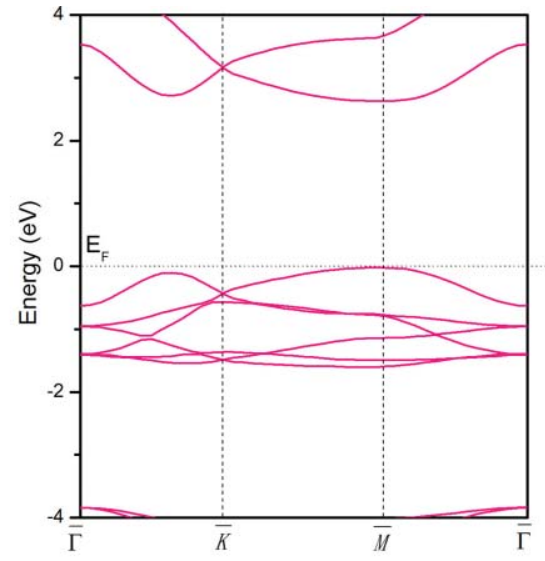

(a)

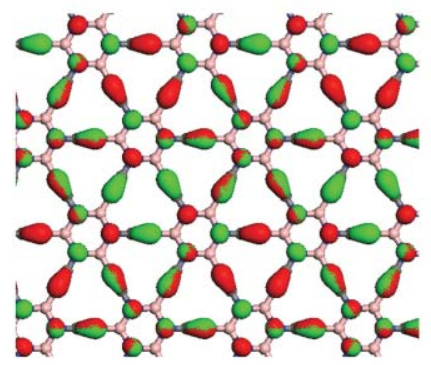

(b)
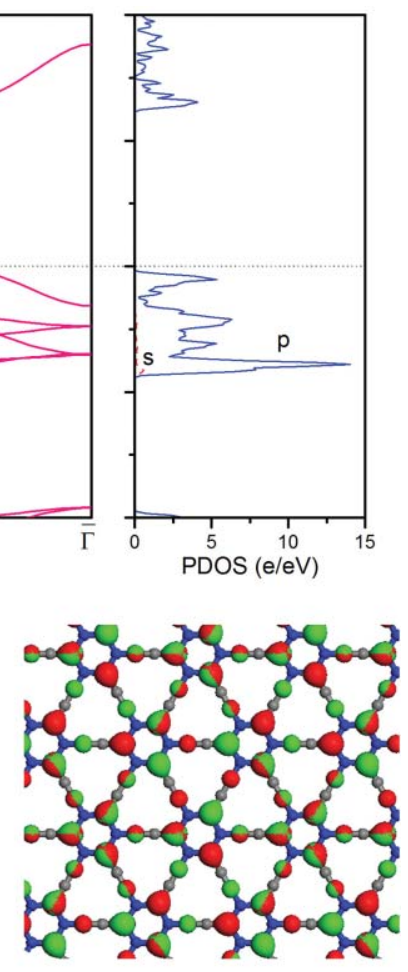

(c)
FIG. 4. Same as in Fig. 2 for BN-yne.

calculated results are given in Fig. 5. There are two competing mechanisms in the studied systems. Longer C-chain can decrease the quantum confinement effect and accordingly decrease the gaps. On the other hand, longer C-chain will further separate the hexagonal rings, which would increase the gap for graphyne-based structure while decreasing the gap in BN-yne-based structure. Thus, the two opposite effects will cancel out to some extent in graphyne-based structure, resulting in minor changes of bandgap with $\mathrm{C}$-chain length, as shown in Fig. 5. Here, the gap changes from $0.47 \mathrm{eV}$ for $n=2$ to $0.52 \mathrm{eV}$ for $n=12, n$ being the chain index (i.e., number of $\mathrm{C}$ atoms in the chain). For BN-yne-based

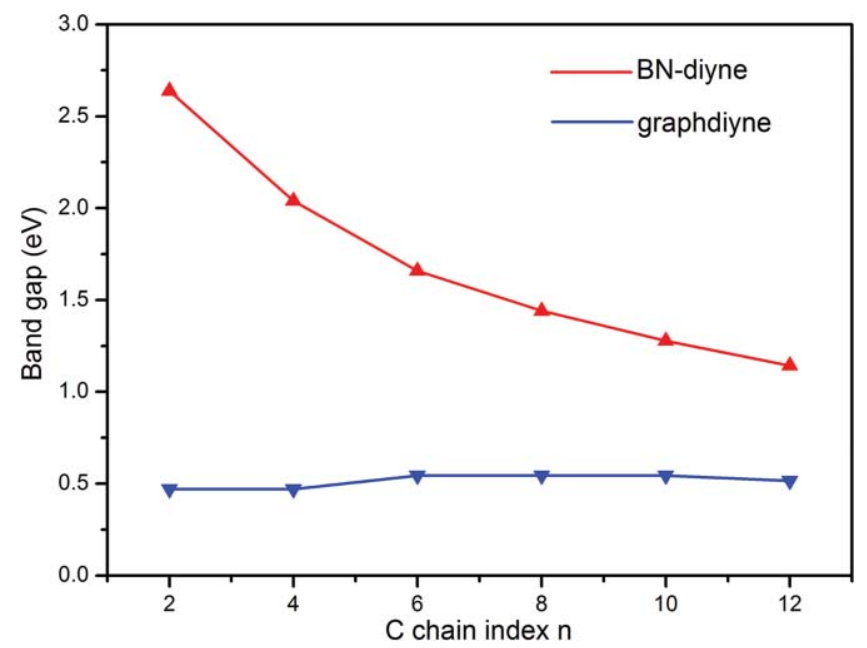

FIG. 5. Bandgap variation of graphyne- and BN-yne-based structures with respect to C-chain length index $n$. structure, these two factors led to a monotonous decrease of bandgap from $2.65 \mathrm{eV}$ for $n=2$ to $1.14 \mathrm{eV}$ for $n=12$. We also studied the systems with different node size $(m)$ and C-chain length $(n)$. For graphyne-based structure, the bandgap changes from $0.47 \mathrm{eV}$ for $(m=6, n=2)$ to 0.62 $\mathrm{eV}$ for $(m=54, n=4)$, while the corresponding changes for $\mathrm{BN}$-yne-based structure is from $2.65 \mathrm{eV}$ to $3.67 \mathrm{eV}$.

In conclusion, motivated by recently synthesized graphdiyne we have studied theoretically the geometric and electronic structures of graphyne and its analog BN-yne. The structures can be viewed as an assembly of hexagonal rings linked via atomic $\mathrm{C}$-chains. They are totally different from the conventional $\mathrm{C}$ structures and open new possibilities for creating $s p$ and $s p^{2}$ bonded carbon by continuously changing the chain length. Main results are summarized as follows: (1) C-chain with even number of $\mathrm{C}$ atoms can lead to $2 \mathrm{D}$ structures, while geometric distortions can be caused by Cchain with odd numbers of $\mathrm{C}$ atoms due to the unpaired electrons. (2) The bond between hexagonal ring and $\mathrm{C}$-chain is a single bond, while single $\mathrm{C}(\mathrm{B}$ or $\mathrm{N})-\mathrm{C}$ and triple $\mathrm{C} \equiv \mathrm{C}$ bond alternations exist in $\mathrm{C}$-chain with even numbers of $\mathrm{C}$ atoms. (3) Both graphyne and BN-yne are direct semiconductors with VBM and CBM located at $\bar{M}$ point of reciprocal space. (4) The HO and LU states of graphyne are contributed by $\pi$ and $\sigma^{*}$ bonding orbitals, respectively. For BN-yne, the $\mathrm{HO}$ is localized on negatively charged $\mathrm{N}$ and triple $\mathrm{C} \equiv \mathrm{C} \pi$ bonds, and the LU is localized on positively charged $\mathrm{B}$ and $\mathrm{C}$ atoms of $\mathrm{C}-\mathrm{N}$ bond. (5) The bandgaps can be modulated by changing the node size $(m)$ and the C-chain length $(n)$, providing more flexibility for energy band engineering. The present study provides insight into the atomic engineering of novel carbon-based 2D structures by using the length of carbon chain as a new parameter.

\section{ACKNOWLEDGMENTS}

This work is partially supported by grants from the National Natural Science Foundation of China (10874007, 10990100), the Foundation of National Laboratory for Infrared Physics, the US National Science Foundation, and the (U.S.) Department of Energy.

${ }^{1}$ K. S. Novoselov, A. K. Geim, S. V. Morozov, D. Jiang, Y. Zhang, S. V. Dubonos, I. V. Grigorieva, and A. A. Firsov, Science 306, 666 (2004).

${ }^{2}$ A. H. Castro Neto, F. Guinea, N. M. R. Peres, K. S. Novoselov, and A. K. Geim, Rev. Mod. Phys. 81, 109 (2009).

${ }^{3}$ X. Li, X. Wang, L. Zhang, S. Lee, and H. Dai, Science 319, 1229 (2008).

${ }^{4}$ C. Jin, H. Lan, L. Peng, K. Suenaga, and S. Iijima, Phys. Rev. Lett. 102, 205501 (2009).

${ }^{5}$ A. Chuvilin, J. C. Meyer, G. Algara-Siller, and U. Kaiser, New J. Phys. 11, 083019 (2009).

${ }^{6}$ J. Bai, X. Zhong, S. Jiang, Y. Huang, and X. Duan, Nat. Nanotechnol. 5, 190 (2010).

${ }^{7}$ M. Bieri, M. Treier, J. Cai, K. Att-Mansour, P. Ruffieux, O. Groning, P. Groning, M. Kastler, R. Rieger, X. Feng, K. Mullen, and R. Fasel, Chem. Commun. (Cambridge) 2009, 6919 (2009).

${ }^{8}$ G. Li, Y. Li, H. Liu, Y. Guo, Y. Lia, and D. Zhu, Chem. Commun. (Cambridge) 46, 3256 (2010).

${ }^{9}$ A. Hirsch, Nature Mater. 9, 868 (2010).

${ }^{10}$ N. Narita, S. Nagai, S. Suzuki, and K. Nakao, Phys. Rev. B 58, 11009 (1998). 
${ }^{11}$ N. Narita, S. Nagai, and S. Suzuki, Phys. Rev. B 64, 245408 (2001).

${ }^{12}$ V. R. Coluci, S. F. Braga, S. B. Legoas, D. S. Galvao, and R. H. Baughman, Phys. Rev. B 68, 035430 (2003).

${ }^{13}$ V. R. Coluci, S. F. Braga, S. B. Legoas, D. S. Galvao, and R. H. Baughman, Nanotechnology 15, 142 (2004).

${ }^{14}$ W. Q. Han, L. Wu, Y. Zhu, K. Watanabe, and T. Taniguchi, Appl. Phys. Lett. 93, 223103 (2008).

${ }^{15}$ C. Zhi, Y. Bando, C. Tang, H. Kuwahara, and D. Golberg, Adv. Mater. 21, 2889 (2009).

${ }^{16}$ L. Ci, L. Song, C. Jin, D. Jariwala, D. Wu, Y. Li, A. Srivastava, Z. F. Wang, K. Storr, L. Balicas, F. Liu, and P. M. Ajayan, Nature Mater. 9, 430 (2010).

${ }^{17}$ J. P. Perdew, K. Burke, and M. Ernzerhof, Phys. Rev. Lett. 77, 3865 (1996).
${ }^{18}$ B. Delley, J. Chem. Phys. 92, 508 (1990).

${ }^{19}$ B. Delley, J. Chem. Phys. 113, 7756 (2000).

${ }^{20}$ H. J. Monkhorst and J. D. Pack, Phys. Rev. B 13, 5188 (1976).

${ }^{21}$ G. Kresse and J. Furthmuller, Phys. Rev. B 54, 11169 (1996).

${ }^{22}$ G. Kresse and J. Joubert, Phys. Rev. B 59, 1758 (1999).

${ }^{23}$ J. Zhou, M. Wu, X. Zhou, and Q. Sun, Appl. Phys. Lett. 95, 103108 (2009).

${ }^{24}$ J. Zhou, Q. Wang, Q. Sun, X. S. Chen, Y. Kawazoe, and P. Jena, Nano Lett. 9, 3867 (2009).

${ }^{25}$ J. Zhou, Q. Wang, Q. Sun, and P. Jena, Phys. Rev. B 81, 085442 (2010).

${ }^{26}$ X. Fan, L. Liu, J. Lin, Z. Shen, and J.-L. Kuo, ACS Nano 3, 3788 (2009).

${ }^{27}$ M. Topsakal, E. Akturk, and S. Ciraci, Phys. Rev. B 79, 115442 (2009). 